\title{
Explorando Modelos Contextuais Híbridos: uma Abordagem Composicional
}

\author{
Roger Machado ${ }^{1}$, Ricardo Almeida ${ }^{1}$, Diórgenes da Rosa ${ }^{1}$, \\ João Lopes ${ }^{2}$, Ana Pernas ${ }^{1}$, Adenauer Yamin ${ }^{1}$ \\ ${ }^{1}$ Universidade Federal de Pelotas (UFPel) \\ ${ }^{2}$ Universidade Federal do Rio Grande do Sul (UFRGS) \\ \{rdsmachado, rbalmeida, diorgenes, marilza, adenauer\}@inf.ufpel.edu.br \\ jlblopeseinf.ufrgs.br
}

\begin{abstract}
The Current proposals of hybrid context modeling brings with it new challenges, among which can cite the form of access and processing of data by applications. Thinking in that, this paper proposes an alternative to solve this problem through a compositional approach to context information in the hybrid models, titled EXEHDA-HM. The proposed approach stands out for the conception of a compositional processing strategy based on rules, which enables the application to combine contextual information stored in different models in a single rule, with the intention to facilitate the identification of situations of interest. To evaluate the approach was developed a case study using a repository composed of a non-relational and triple model, the results achieved stimulate continuation of the research.
\end{abstract}

Resumo. As atuais propostas de modelagem híbrida de contexto trazem consigo novos desafios, dentre os quais pode-se citar a forma de acesso e processamento destes contextos por parte das aplicações. Neste enfoque, o presente trabalho propõe uma alternativa para solucionar este problema por meio de uma abordagem composicional de informações contextuais em modelos híbridos, intitulada EXEHDA-HM. A abordagem proposta se destaca pela concepção de uma estratégia de processamento composicional baseada em regras, a qual possibilita à aplicação combinar informações contextuais armazenadas em diferentes modelos em uma única regra, tendo como intuito facilitar a identificação de situações de interesse. Para avaliação da abordagem foi desenvolvido um estudo de caso utilizando um repositório composto de um modelo não relacional e de triplas, os resultados atingidos estimulam a continuidade da pesquisa.

\section{Introdução}

Ao se construir e executar aplicações ubíquas cientes de contexto há uma série de funcionalidades que devem ser providas, envolvendo desde a aquisição de informações contextuais, a partir de fontes heterogêneas e distribuídas, até a representação dessas informações, seu processamento, armazenamento e a realização de inferências para seu uso em tomadas de decisão [Bellavista et al. 2012].

Conhecer o contexto em que ocorre uma interação mostra-se uma tarefa complexa em sistemas computacionais. A construção de sistemas cientes de contexto exige, 
inicialmente, a definição do que considerar como contexto, onde este se aplica e que informações são necessárias para descrevê-lo. É preciso viabilizar formas de realizar a aquisição do contexto de forma mais automática possível. Após a coleta de dados brutos a partir do sensoriamento, tornam-se necessários mecanismos de processamento de contexto que tratem as informações coletadas, produzindo informações contextualizadas [Bauer et al. 2014].

Atualmente, as aplicações cientes de contexto tendem a tratar com dados de diferentes naturezas, onde os mesmos podem ser modelados utilizando diversas abordagens. Nesta perspectiva se observa uma tendência na utilização de repositórios com diferentes modelos de armazenamento, chamados de modelos híbridos [Kotenko et al. 2013] [Perera et al. 2014], pois muitas vezes a utilização de somente um modelo para armazenamento das informações contextuais acaba não sendo o ideal, por questões ligadas a desempenho, utilização de disco, entre outras [Carvalho 2014].

O crescente uso de modelos híbridos traz consigo uma preocupação relacionada a forma com que as aplicações realizam o acesso aos dados. Tendo em vista o acesso e processamento de dados provenientes de diferentes modelos a proposta desenvolvida, denominada EXEHDA-HM (Execution Environment for Highly Distributed ApplicationsHybrid Modelling), tem como premissa oferecer suporte à utilização de modelagem híbrida de contexto. Para tanto a EXEHDA-HM provê: repositório único, que permite o armazenamento das informações contextuais em diferentes modelos; uma estratégia de processamento composicional de contexto, a qual possui como diferencial a capacidade de combinação das informações presentes nos diferentes modelos de contexto utilizados. A abordagem proposta é integrada ao middleware EXEHDA [Lopes et al. 2014], contribuindo com o seu Subsistema de Adaptação e Reconhecimento de Contexto.

Este artigo está dividido da seguinte forma: a Seção 2 discute a Ciência de Contexto e as etapas envolvidas para a sua obtenção; a Seção 3 apresenta a abordagem EXEHDA-HM, mostrando a sua arquitetura e suas principais funcionalidades; a Seção 4 explora um estudo de caso, o qual demonstra o funcionamento da abordagem proposta; a Seção 5 descreve os trabalhos relacionados; e, por fim, a Seção 6 discute as considerações finais e os trabalhos futuros.

\section{Ciência de Contexto}

A ciência de contexto é um dos requisitos para obtenção da computação ubíqua, sendo vista como a capacidade de um sistema em usar o contexto para prover serviços e/ou informações relevantes para o usuário [Dey 2001] [Knappmeyer et al. 2013].

De forma resumida, a construção de um sistema ciente de contexto se dá por meio de três etapas: aquisição, a qual refere-se ao processo de monitorar e capturar informações contextuais; modelagem, que consiste na concepção de um modelo de entidades do mundo real, suas propriedades, estado de seu ambiente e situações que podem ser usadas como referência para a aquisição, interpretação e raciocínio de informações contextuais [Knappmeyer et al. 2013]; e processamento, o qual pode ser definido como um mecanismo de raciocínio para inferir novos conhecimentos e melhorar a compreensão dos contextos adquiridos [Neto et al. 2015].

Com relação a estas etapas, observa-se nos trabalhos a adoção de diferentes es- 
tratégias provenientes de campos da inteligência artificial e de sistemas baseados em conhecimento, sendo a estratégia baseada em regras a mais utilizada [Perera et al. 2014].

Já relacionado à etapa de modelagem de contexto, diversas abordagens são encontradas na literatura, cada uma com suas vantagens e desvantagens, não havendo ainda consenso a respeito de qual seria a ideal. Dadas as diferentes necessidades de representação de dados em aplicações ubíquas, abordagens híbridas tem sido consideradas promissoras, pois combinam duas ou mais formas de modelagem com variados níveis de interpretação e diferentes aspectos de uso.

Com a utilização de modelos híbridos de contexto novos desafios se mostram presentes, dentre os quais destacam-se: (i) o gerenciamento das informações contextuais; (ii) o armazenamento em diferentes modelos, proporcionando a persistência das informações contextuais; e (iii) o processamento destas informações, já que com o emprego de modelos híbridos as aplicações podem ter os contextos armazenados em diferentes modelos e, muitas vezes, é importante poder combinar estas informações visando facilitar a identificação das situações de interesse.

\section{EXEHDA-HM}

Tendo em vista os desafios de utilização das abordagens híbridas, o presente trabalho propõe a abordagem EXEHDA-HM, a qual possibilita o emprego de modelos híbridos de contexto, onde é concebido um repositório híbrido para armazenamento das informações contextuais, provendo as facilidades particulares de cada modelo utilizado e uma estratégia para processamento dos contextos adquiridos com base em regras composicionais. As regras composicionais possuem como diferencial a capacidade de combinar em uma mesma regra as informações presentes nos diferentes modelos de armazenamento, onde para isso são utilizadas tags de marcação na regra criada, sendo estas tags substituídas pelas informações que serão buscadas no modelo desejado.

A arquitetura da abordagem EXEHDA-HM é apresentada na Figura 1, onde pode ser visualizada na parte superior da Figura a camada de processamento, responsável pelo tratamento das regras composicionais, e o repositório desenvolvido, intitulado de RHIC (Repositório Híbrido de Informações Contextuais), o qual é dividido em duas camadas: a camada de serviço, responsável por disponibilizar métodos que permitem o acesso às informações presentes no repositório; e a camada de armazenamento, para provimento de formas diferenciadas de armazenamento.

A abordagem proposta foi integrada ao middleware EXEHDA, de forma a se beneficiar do suporte à aquisição, processamento e armazenamento de informações contextuais, assim como da arquitetura distribuída do middleware, características oportunas às funcionalidades da EXEHDA-HM. Para realizar esta integração modificações se fizeram necessárias na arquitetura de software do EXEHDA, as principais foram realizadas no Subsistema de Adaptação e Reconhecimento de Contexto, sendo garantida a interface desse subsistema com os outros existentes no middleware.

A seguir são apresentadas as três camadas presentes na EXEHDA-HM.

\subsection{Camada de Armazenamento}

Como também pode ser observado na Figura 1, o RHIC provê um repositório com três modelos de armazenamento: 


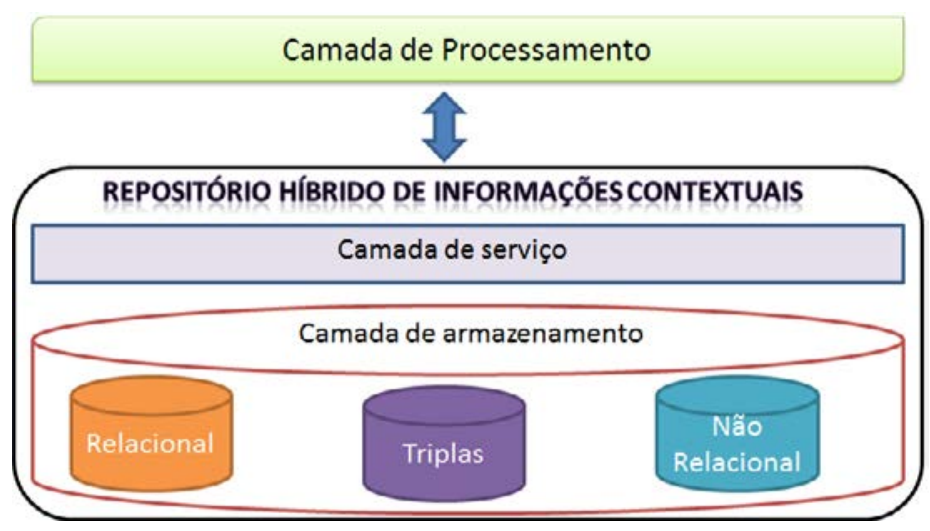

Figura 1. Arquitetura da EXEHDA-HM

- modelo relacional: modelo já utilizado pelo RIC (Repositório de Informações Contextuais) do EXEHDA para armazenar informações contextuais. A sua utilização é mantida, pois se comporta de forma satisfatória para diversas situações, como por exemplo, armazenar os dados dos sensores que estão sendo monitorados por cada servidor de borda presente no ambiente ubíquo, onde no repositório é mantida a relação do servidor de borda com o sensor, facilitando o acesso à esta informação como também a sua modificação caso seja necessário;

- modelo de triplas (sujeito, predicado, objeto): empregado para ser utilizado na persistência de ontologias. Com a inserção do modelo de armazenamento de dados no formato de triplas, espera-se prover facilidades similares às experimentadas nos sistemas gerenciadores dos modelos relacionais, onde o acesso e a manipulação das informações presentes nas ontologias é feito de forma automatizada. Exemplos de manipulação seriam a inserção de novas instâncias e a criação de novas classes no modelo;

- modelo não relacional: utilizado tendo como premissa o suporte ao tratamento de grandes volumes de dados. Atualmente, tem surgido um aumento na quantidade e variedade de dados de contexto que devem ser tratados pelo middleware EXEHDA, como por exemplo, arquivos de log de aplicações, os quais são analisados visando, dentre outras finalidades, melhorar a segurança do ambiente computacional. Para implementação deste modelo foi escolhida a categoria orientada a documentos, a qual possui como característica ignorar campos vazios, o que otimiza o espaço em disco utilizado, sendo recomendada para o tratamento de dados semiestruturados, os quais possuem uma estrutura irregular, em que não existe um esquema padrão dos dados [Pramod J Sadalage 2013].

$\mathrm{Na}$ abordagem proposta fica a cargo da aplicação determinar onde prefere armazenar as informações contextuais, sendo indicada a utilização do modelo relacional para dados que possuem relação entre eles, e assim, aproveitando-se das características do modelo. O modelo de triplas para armazenar as ontologias, de forma a propiciar facilidades para o tratamento das informações presentes nas mesmas. E recomenda-se o armazenamento no modelo não relacional quando é necessário o tratamento de grande volume de dados e/ou ainda, quando os dados armazenados possuem variedade de formatos. 


\subsection{Camada de Serviço}

A camada de serviço desenvolvida para o RHIC é responsável pela disponibilização de métodos que permitem a busca, inserção e exclusão de informações contextuais na camada de armazenamento.

As consultas realizadas na camada de armazenamento podem ser executadas diretamente pela interface web disponibilizada para o gerenciamento da EXEHDA-HM, onde podem ser visualizadas as informações presentes no repositório, ou utilizando o mecanismo de gerenciamento das informações contextuais, o qual permite o acesso às informações de forma que as aplicações possam realizar as consultas em nível de programação utilizando o método de consultas oferecido pelo mecanismo na linguagem Java.

As ontologias são processadas antes de serem armazenadas no repositório de triplas, desta forma, no momento em que as aplicações desejarem consultar as informações presentes no modelo ontológico, o processo de inferência já estará realizado, podendo resultar em uma melhora do tempo de resposta do sistema, já que o processo de inferência pode se tornar lento dependendo do tamanho da ontologia a ser processada.

\subsection{Camada de Processamento}

A EXEHDA-HM oferece o suporte ao processamento das informações contextuais utilizando uma estratégia baseada em regras composicionais. Para permitir à aplicação a composição das regras usando dados dos diferentes modelos, as regras definidas devem utilizar tags de marcação que comecem com o símbolo “\#”, o qual é substituído pela consulta responsável por buscar esta informação no RHIC.

A camada de processamento realiza a execução da regra composicional seguindo um fluxo específico de execução, conforme pode ser visualizado na Figura 2. Primeiramente é buscada a regra desejada no RHIC, após, são identificadas as consultas auxiliares que devem ser realizadas, tendo como intuito buscar os dados necessários no RHIC para substituição das tags de marcação presentes na regra buscada. Posteriormente, com o retorno das consultas auxiliares, a regra é composta pelos valores necessários e sua avaliação é realizada, tendo como retorno o comando desejado a ser executado, cujo identificador encontra-se na própria regra. Com isso é necessário buscar o comando desejado no RHIC e, então, executar o comando retornado.

Destaca-se que as regras composicionais podem ser compostas de dados armazenados nos diferentes modelos, tornando a consulta mais expressiva e, em consequência, facilitando à aplicação identificar situações de interesse. Caracteriza-se ainda a melhora na atualização e manutenção das regras criadas, pois caso mais de uma aplicação utilize as mesmas regras só é necessário atualizar seu conteúdo em um determinado campo e todas estarão atualizadas. Além disso, os comandos que determinam as ações decorrentes das situações não são definidos nas regras, mas em uma tabela do RHIC, o que facilita a reutilização e manutenção dos mesmos.

\section{Cenário de Uso}

Durante os últimos anos, o Departamento de Segurança da Informação e Comunicações (DSIC) da Presidência da República do Brasil estabeleceu diversos decretos relacionados à Segurança da Informação e Comunicações no âmbito da Administração Pública 


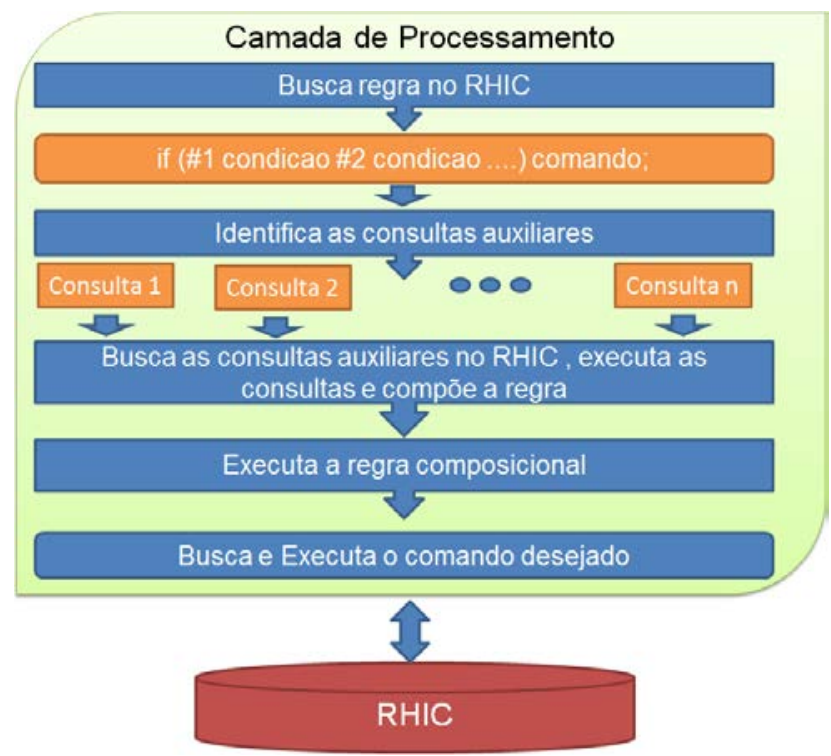

Figura 2. Fluxo de execução da regra composicional

Federal (APF). Além disso, o DSIC desenvolveu algumas normas complementares com diretrizes para ações a serem desenvolvidas pela APF, sendo que algumas destas normas referenciam a ABNT NBR ISO/IEC 27001:2013 e a ABNT NBR ISO/IEC 27002:2013.

Tendo isto em vista, que um dos requisitos a ser cumprido pelas instituições Federais é a elaboração da Política de Segurança da Informação e Comunicações (POSIC) [BRASIL 2000]. Sabe-se que um dos desafios relacionados à POSIC é a sua implementação e, mais especificamente, a verificação da sua conformidade. Por exemplo, definir como verificar se os usuários estão em conformidade com a política de senhas (documento geralmente derivado da POSIC).

Desta forma, este estudo de caso explora a ISO 27002 que apresenta um código de prática para a gestão da segurança da informação. Mais especificamente, este estudo de caso demonstra a utilização da abordagem EXEHDA-HM empregando o modelo não relacional e o modelo de triplas, onde são combinadas as informações presentes nos dois modelos com a aplicação da estratégia de processamento composicional.

Para implementação deste estudo de caso foi utilizada uma ontologia baseada em [Alcázar and Fenz 2012], a qual é responsável por mapear a estrutura da ISO 27002, incluindo seus controles, ativos, ameaças e vulnerabilidades. Nesta ontologia foram adicionadas novas classes de forma a facilitar o acesso às informações de interesse, sendo que optou-se por adicionar como subclasses as diretrizes relacionadas a "password policy", tais como, "complexity requirements" e "clear text'. A ontologia foi armazenada no modelo de triplas presente no RHIC.

Como fonte para a aquisição de contexto, foi explorado o monitoramento dos logs do Web Application Firewall modsecurity [ModSecurity 2016] que foi devidamente configurado para identificar quebras na política de senhas de aplicações web. Após o tratamento do log é realizada a identificação da situação de interesse caracterizada pela mensagem "password does meet complexity requirements", e por último a situação identificada é armazenada no modelo não relacional do RHIC, já que as situações possuem 
um formato semiestruturado.

Com a situação já identificada e armazenada no RHIC, pode ser utilizada a estratégia de processamento composicional conforme apresentado na Figura 3, com a execução da regra if(\#3 \&\& \#4) “ $b$ ". A tag \#3 é substituída pela consulta find (eq ("subcategory", "ComplexityRequirements")) a qual busca no modelo não relacional as situações com o atributo "subcategory" igual a "ComplexityRequirements". A tag \#4 representa a consulta SPARQL [W3C 2008] que é apresentada na Figura 4, a qual busca os controles a serem sugeridos que estão relacionados a ComplexityRequirements de acordo com a ontologia da ISO 27002 e as propriedades controlStatement, implementationGuidance, otherInformation onde estão inseridas as informações a respeito de cada controle que foi sugerido.

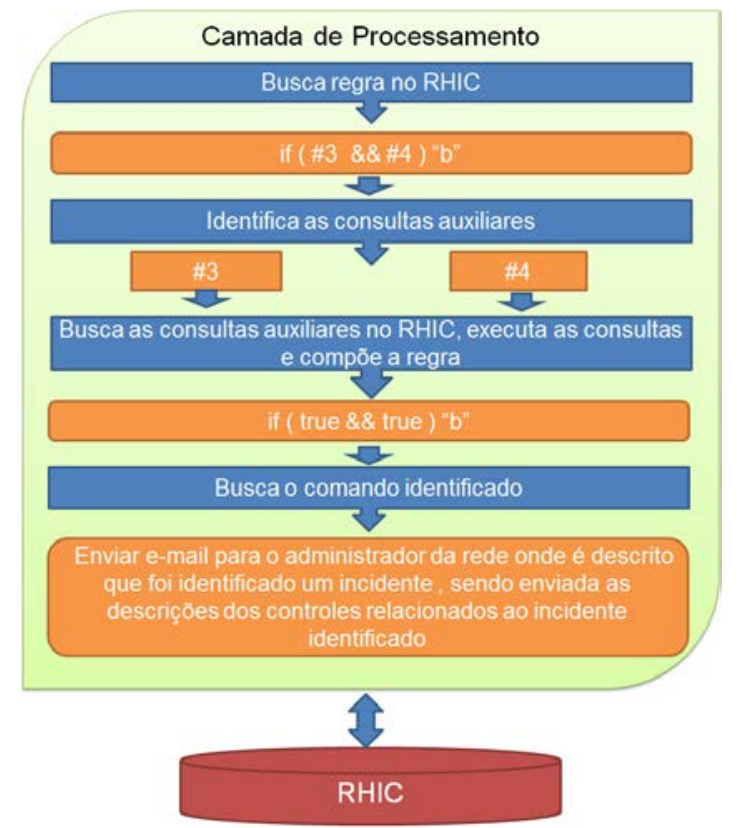

Figura 3. Funcionamento da regra para o reforço de políticas de senha

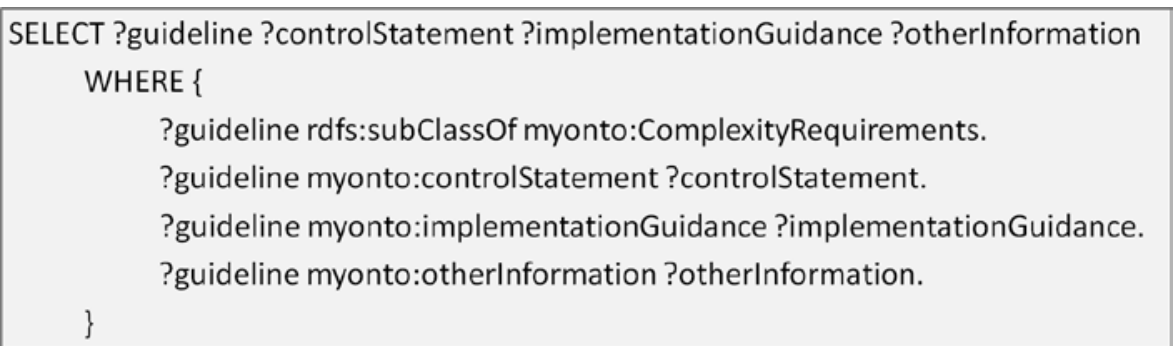

Figura 4. Consulta referente à busca pelos controles sugeridos relacionados à situação "complexity requirements"

Como condição a ser executada na regra composicional, foi utilizado o método que verifica se foi retornado algum resultado na consulta, o qual retorna "true", caso positivo, e "false", caso não tenha nenhum retorno. O comando previamente configurado para ser executado, caso seja detectada a situação, é representado por "b", sendo este referente ao envio de e-mail para os analistas de segurança da informação. Este e-mail é composto de 
uma mensagem de notificação de incidente, junto aos controles que constam na ontologia da ISO 27002 que servirão como sugestões de melhorias a serem empregadas, sendo os analistas responsáveis por avaliar a necessidade de tomada de ações para reforçar a política de senhas.

\section{Trabalhos Relacionados}

[Ejigu et al. 2007] propôs um modelo híbrido de contexto utilizando o modelo relacional e o baseado em ontologia para o middleware CoCA (Collaborative Context-Aware Service Platform), o qual realiza as tarefas de representação, raciocínio, agregação e interpretação de dados de contexto. As ações e decisões são realizadas com base nos dados de contexto adquiridos, e a plataforma suporta a colaboração e o compartilhamento de recursos computacionais entre os dispositivos.

Em [Kotenko et al. 2013] é apresentada uma abordagem de modelo híbrido de contexto para sistemas SIEM (Security Information and Events Management) que combina os modelos relacionais, baseados em XML e em ontologias. Os autores justificam que a representação relacional não permite especificar uma consulta parametrizada, por exemplo, com os nomes dos produtos e versões, para a análise de vulnerabilidades e processá-las no programa, o que torna este processo demorado. Com isso, uma solução alternativa na representação de dados em sistemas de processamento de informações com estruturas complexas de dados (tais como sistemas SIEM) é uma abordagem ontológica, o que facilita as expressões de relações complexas entre entidades e possibilita a formulação de conceitos e de relacionamentos envolvidos.

Em [VANATHI 2013] é apresentado um sistema ciente de contexto que baseia-se em uma modelagem híbrida de contexto, a qual combina os modelos objeto relacional e ontológico. O modelo objeto relacional é criado a partir dos dados de contexto estáticos, e são atualizados em tempo de execução com os novos dados de contexto capturados. O modelo baseado em ontologia Context-Onto é composto de três componentes básicos: o esquema da ontologia, dados de contexto estático e dados de contexto dinâmicos. O esquema da ontologia representa as hierarquias de conceito, conceito de relações, axiomas e informações de metadados, que representam o conhecimento.

Analisando a etapa de processamento dos trabalhos relacionados, apesar de utilizarem diferentes estratégias para modelagem de contexto, o acesso aos modelos é realizado de forma individual, não possibilitando a combinação das informações presentes nos diferentes modelos. Por outro lado, a EXEHDA-HM foi desenvolvida de forma a prover uma estratégia de regras composicionais que permita à aplicação combinar os contextos de diferentes modelos, viabilizando a utilização de forma combinada das informações contextuais.

Destaca-se ainda a utilização de diferentes formas de armazenamento da EXEHDA-HM, a qual possibilita explorar as vantagens de cada um dos três modelos de armazenamento: relacional; de triplas; e não relacional.

\section{Considerações Finais}

Neste trabalho foram tratados os desafios enfrentados pelas aplicações cientes de contexto com o emprego de modelos híbridos, onde destaca-se a ausência de uma estratégia 
que permita utilizar os contextos presentes nos diferentes modelos utilizados. Com estes desafios em vista, as seguintes contribuições foram alcançadas com o desenvolvimento deste trabalho: (i) a concepção de uma abordagem com suporte a modelagem híbrida de contexto; (ii) uma camada de armazenamento que provê a persistência em três modelos distintos; (iii) uma camada de serviço que permite o acesso as informações contextuais por parte das aplicações cientes de contexto; e (iv) a concepção de uma estratégia de processamento composicional de contexto, a qual possui como diferencial a possibilidade de combinar dados presentes nos diferentes modelos de armazenamento utilizados.

Por meio da EXEHDA-HM foi realizada a concepção de um novo repositório para armazenamento de informações contextuais, o RHIC, dividido em duas camadas:

- camada de serviço: responsável por gerenciar as informações contextuais presentes na camada de armazenamento. Esta camada disponibilizou métodos para consulta, inserção e remoção de dados na camada de armazenamento. Foram disponibilizados métodos para tratar as informações contextuais nos três modelos de armazenamento presentes no RHIC;

- camada de armazenamento: distinguiu-se pela utilização de três modelos para o armazenamento de informações contextuais tratadas pelo middleware EXEHDA: relacional, modelo que vinha sendo utilizado pelo middleware; não relacional, ofereceu o suporte ao armazenamento eficiente para dados semiestruturados; e de triplas, que foi escolhido para realizar o armazenamento das informações contextuais presentes nas ontologias, oferecendo mecanismos que facilitam o acesso à estas informações.

Além da criação do RHIC, a abordagem EXEHDA-HM possui como principal contribuição a camada de processamento desenvolvida, onde se utiliza de uma estratégia baseada em regras composicionais, permitindo combinar nas regras criadas dados de diferentes modelos de armazenamento. Com a utilização da abordagem EXEHDA-HM aumenta-se a flexibilidade e a possibilidade de expressividade da aplicação, permitindo que sejam utilizados em uma mesma regra dados armazenados nos diferentes modelos, melhorando a identificação das situações de interesse das aplicações.

O estudo de caso explorou a aplicação da EXEHDA-HM em um escopo de atual relevância devido ao aumento das preocupações com segurança da informação nas instituições. Neste cenário foi explorado um problema comum e de considerável risco as instituições, onde a solução proposta possibilitou a integração das situações armazenadas no modelo não-relacional com as informações presentes na ontologia da ISO 27002, tendo como resultado a indicação de controles de segurança aos analistas.

Dentre os aspectos levantados para continuidade do trabalho, podem ser citados: (i) desenvolver interfaces que utilizem técnicas de visualização de dados, com o intuito de facilitar a interpretação das informações contextuais armazenadas e das situações identificadas; (ii) aplicar a abordagem EXEHDA-HM em diferentes cenários de utilização, por exemplo, em ambientes educacionais, os quais costumam utilizar tanto os modelos baseados em ontologias quanto os modelos relacionais.

\section{Referências}

Alcázar, F. and Fenz, S. (2012). Mapping iso 27002 into security ontology. Treball final de grau - Universitat Politècnica de Catalunya. Escola d'Enginyeria de Telecomunicació 
i Aeroespacial de Castelldefels.

Bauer, J. S., Newman, M. W., and Kientz, J. A. (2014). Thinking about context: Design practices for information architecture with context-aware systems. In iConference 2014 Proceedings, pages 398-411.

Bellavista, P., Corradi, A., Fanelli, M., and Foschini, L. (2012). A survey of context data distribution for mobile ubiquitous systems. ACM Comput. Surv., 44(4):24:1-24:45.

BRASIL (2000). Decreto lei $n^{0} 3505$ - institui a política de segurança da informação nos órgãos e entidades da administração pública federal. Diário Oficial da União.

Carvalho, A. G. (2014). Interface nosql integrada a banco relacional para gerenciamento de dados em nuvem privada. Monografia bacharelado em engenharia da computação, Centro Universitário de Brasília Faculdade de Tecnologia e Ciências Sociais Aplicadas.

Dey, A. K. (2001). Understanding and using context. Personal Ubiquitous Computing, $5(1): 4-7$.

Ejigu, D., Scuturici, M., and Brunie, L. (2007). Coca: A collaborative context-aware service platform for pervasive computing. In Information Technology, 2007. ITNG '07. Fourth International Conference on, pages 297-302.

Knappmeyer, M., Kiani, S., Reetz, E., Baker, N., and Tonjes, R. (2013). Survey of context provisioning middleware. Communications Surveys Tutorials, IEEE, 15(3):14921519.

Kotenko, I., Polubelova, O., Chechulin, A., and Saenko, I. (2013). Design and implementation of a hybrid ontological-relational data repository for siem systems. Future Internet - Special Issue Security of Systems and Software Resiliency.

Lopes, J., Souza, R., Geyer, C., Costa, C., Barbosa, J., Pernas, A., and Yamin, A. (2014). A middleware architecture for dynamic adaptation in ubiquitous computing. Journal of Universal Computer Science, 20(9):1327-1351. http : / / www • jucs . org/ jucs_ 20_9/a_middleware_architecture_for.

ModSecurity (2016). Acesso em 26 de maio de 2016. Disponível em: < https : / / www . modsecurity.org/>.

Neto, A., Azevedo, B., Bouefleuer, R., Lima, J., and Augustin, I. (2015). Modelo para predição de ações e inferência de situações de risco em ambientes sensíveis ao contexto. In Simpósio Brasileiro de Computação Ubíqua e Pervasiva.

Perera, C., Zaslavsky, A., Christen, P., and Georgakopoulos, D. (2014). Context aware computing for the internet of things: A survey. Communications Surveys Tutorials, IEEE, 16(1):414-454.

Pramod J Sadalage, M. F. (2013). NoSQL Essencial, Um Guia Conciso para o Mundo Emergente da Persistência Poliglota. Novatec.

VANATHI, B. (2013). Context Management Using Ontology and Object Relational Database Management System. Doctor of philosophy, Faculty of Information and Communication Engineering Anna University, Chennai.

W3C (2008). Acesso em 26 de maio de 2016. Disponível em: <https : / / www.w3 . org/TR/rdf-sparql-query/>. 